\title{
A Theoretical Explanation for Rock Core Disking in Triaxial Unloading Test by Considering Local Tensile Stress
}

\author{
Houxu HUANG ${ }^{1}$, Pengxian FAN ${ }^{1}$, Jie LI ${ }^{1,2}$, Mingyang WANG ${ }^{1,2}$, \\ and Xiaoli RONG $^{1}$ \\ ${ }^{1}$ State Key Laboratory of Disaster Prevention and Mitigation of Explosion \\ and Impact, PLA University of Science and Technology, Nanjing, China; \\ e-mail: wuhanhp14315@163.com \\ ${ }^{2}$ School of Mechanical Engineering, Nanjing University of Science and Technology, \\ Nanjing, China
}

\begin{abstract}
Rock is a typical inhomogeneous material with a large number of flaws in different scales; the stress field of the rock in its elastic state consists of two parts: the elastic stress, which distributes uniformly in the entire region; and an additional stress, which only exists around the flaws. Theoretical expressions of the additional stress and local stress are derived based on the Maxwell model. Core disking which takes place under the condition that the axial stress is rapidly reduced while the confining pressure is kept unchanged is explained with a new method. Unloading duration's effect on core disking is analyzed. A new criterion for core disking is presented based on attributing the core disking to the result of the exceedance of local tensile stress over the tensile strength. Based on our theoretical analysis and the conclusions from published resources, core disking is most likely to occur if the maximum principal stress is more than five to six times the tensile strength.
\end{abstract}

Key words: flaws, additional stress, local stress, new criterion, core disking. 


\section{INTRODUCTION}

Rock core disking is often observed in high stress regions. Many researchers tried to explain this phenomenon; approaches such as site observations (Lempp and Muhlhaus 1985, Li and Schmitt 1998), laboratory tests (Bauch and Lempp 2004) and numerical analysis (Corthesy and Leite 2008, Zhu et al. 2014) have all been adopted to investigate the mechanism of rock core disking. Jaeger and Cook (1963) reported that the maximum principal stress corresponding to core disking is less than the uniaxial compressive strength of the rock. Therefore, they attributed core disking to the shear stress. But the phenomenon that there is no shearing trace on the new faces indicates that core disking should be a tensile failure. Obert and Stephenson (1965) in the laboratory experiment of conventional triaxial compression test showed that core disking occurs if the radial stress exceeds half of the compressive strength of the rock. Thus, they also attributed core disking to the shear stress, but they also found that the newly generated faces are more likely to be created by the tensile stress. In addition, Ishida and Saito (1995) reported that the existing core disking prediction models, which are based on shear stress criterion, do not work well. Many other researchers also showed that the core disking is fundamentally a tensile failure phenomenon (Stacey 1982, Matsuki et al. 2004, Almeida et al. 2006, Qi and Qian 2009, Lim and Martin 2010).

Based on the extended Hooke's law and the effective tensile stress, Bauch and Lempp (2004) found that the effective tensile strain criterion for rock core disking, which was proposed by Stacey (1982), can be expressed as

$$
\varepsilon_{c}=\varepsilon_{3}=\varepsilon_{\text {axial }}=\frac{\sigma_{3}^{\prime}}{E}=\frac{\sigma_{3}-v\left(\sigma_{1}+\sigma_{2}\right)}{E}
$$

where $\sigma_{1}, \sigma_{2}$, and $\sigma_{3}$ are principal stresses, $\sigma_{3}^{\prime}$ is the effective tensile stress, $\varepsilon_{c}, \varepsilon_{3}$, and $\varepsilon_{\text {axial }}$ are the critical extensile strain, minor strain and axial strain, respectively. Based on the triaxial extension tests with the cylindrical specimens, Bauch and Lempp (2004) pointed out that the rock core disking is time dependent and cannot be explained by the critical strain criterion. They found that in the abrupt mode (with the confining pressure kept constant and the axial stress reduced rapidly), the core disking can take place even if the axial strain is far less than $0.1 \%$, but when the reduction rate of the axial stress is low (approximately quasi-static), the core disking cannot be observed even if the axial strain exceeds $1.5 \%$.

The previous studies have made significant contributions to our understanding of the cause for rock core disking; we have learned that both the 
stress state and the unloading duration have direct effects on rock core disking, but the unloading duration's effect on rock core disking lacks study.

In fact, the rock is an inhomogeneous material and contains a large number of flaws in different scales; the mechanical properties of the flaw are different from those of the elastic medium. According to Maxwell model (Rodionov and Sizov 1982, 1989), both in the loading and unloading processes, the flaw will cause an additional stress and there are additional stress's concentration and relaxation in the rock, i.e., the additional stress is time dependent. In addition, the tensile stress which causes the rock core disking is always regarded as a synthesis of the principal stresses, but little attention is paid to the additional stress's effect on rock core disking. What is more, during the unloading of the axial stress, when the core disking occurs, the newly generated faces are almost perpendicular to the axial direction of the cylindrical specimen, which means there must be tensile stress in the axial direction, but this phenomenon is hard to explain with the principal stress. Thus, to explain the rock core disking in a new way seems meaningful and necessary.

In this paper, the expressions for additional stress and local stress are derived; unloading duration's effect on core disking is analyzed. A new criterion, which is based on attributing core disking to the local tensile stress exceedance over the tensile strength, is established; the stress condition for rock core disking is predicted. In the end, the theoretical prediction is tested by means of both the laboratory test and the statistical data from the published resources.

\section{ADDITIONAL STRESSES IN AN ELASTIC ROCK}

There is a large number of flaws in the rock and the distribution of stress field in the rock is non-uniform; stress fluctuations occur around flaws both in the loading and unloading processes. As shown in Fig. 1, the specimen consists of an elastic medium and a large number of flaws with different scales.

In the following analysis, several concepts are introduced, including: the additional stress $\Delta \sigma_{i j}$ which is caused by the flaws and exists in regions such as region I (as shown in Fig. 1b); the elastic stress $\sigma_{i j}^{e}$, which characterizes the stress distributed uniformly in the entire region and can be obtained by using the Hooke's law; and the local stress $\sigma_{i j}^{\text {loc }}$, which characterizes the stress existing in regions such as region I and is the superposition of the elastic stress $\sigma_{i j}^{e}$ and the additional stress $\Delta \sigma_{i j}$. Therefore, the expression of the local stress $\sigma_{i j}^{\text {loc }}$ can be written as 


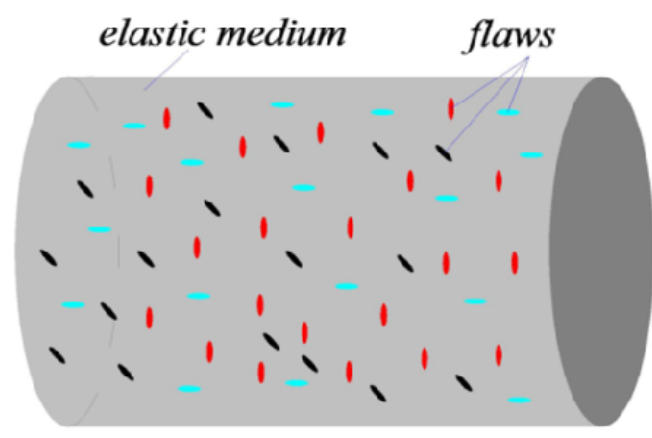

(a)

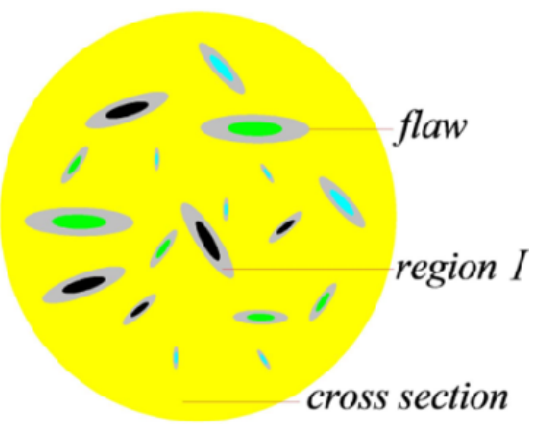

(b)

Fig. 1: (a) Rock specimen, which can be regarded as a composition of two parts, the elastic medium and the flaws with different scales; (b) Cross section perpendicular to the axial direction of the specimen: the stress field in region I is influenced by the flaw and there is stress fluctuation within region I; besides, the scope of regions corresponding to different flaws are not the same.

$$
\sigma_{i j}^{\mathrm{loc}}=\sigma_{i j}^{e}+\Delta \sigma_{i j} .
$$

There is additional stress $\Delta \sigma_{i j}$ which is caused by the flaw both in the loading and unloading processes. The elastic stress is not time dependent, but the concentration and relaxation of the additional stress are time dependent; thus, we can deduce that the stresses in the regions which are influenced by the flaws are time dependent. According to Maxwell model, the evolution of the additional stress can be expressed as (Rodionov and Sizov 1982, 1989)

$$
\frac{\mathrm{d} \Delta \sigma_{i j}}{\mathrm{~d} t}=k \rho c_{s}^{2} \dot{e}_{i j}-\frac{\eta}{l} \Delta \sigma_{i j},
$$

where $\eta$ is the stress relaxation rate, $l$ is the flaw scale, $\rho$ is the density of the medium, $c_{s}$ is the velocity of the shear wave, $\dot{e}_{i j}$ is the corresponding strain rate component, $k$ is the stress concentration coefficient. The first term on the right-hand side of Eq. 3 describes the elastic loading, while the second term on the right-hand side of Eq. 3 depicts the stress relaxation.

According to Eq. 3 the solution of $\Delta \sigma_{i j}$ is obtained

$$
\Delta \sigma_{i j}=C e^{-\frac{\eta}{l} t}+\frac{k \rho c_{s}^{2} \dot{e}_{i j}}{\eta}
$$

where $C$ is an integration constant and depends on the boundary conditions. 


\section{CORE DISKING IN TRIAXIAL UNLOADING TEST AND ITS EXPLANATION}

According to Bauch and Lempp (2004), core disking is most likely to take place in an abrupt mode; therefore, the mechanical model and the unloading mode which are adopted in our analysis are as follows.

Figure 2 shows that, before unloading, the specimen is in an initial hydrostatic stress state, $\sigma_{0}$, where $\sigma_{a}=\sigma_{r}=\sigma_{0}$. During the unloading process, the axial stress will rapidly decrease from $\sigma_{a}$ to zero, while the confining pressure is kept constant; the total axial strain and unloading duration are, respectively $\varepsilon_{0}$ and $T_{0}$, the axial strain rate $\dot{\varepsilon}_{z z}^{u}$ in this process can be expressed as $\dot{\varepsilon}_{z z}^{u}=\varepsilon_{0} / T_{0}$.

When $0 \leq t \leq T_{0}$, the axial strain $\varepsilon_{z z}^{u}(t)$ and the axial stress $\sigma_{z z}^{u}(t)$ can be expressed as

$$
\left\{\begin{array}{l}
\varepsilon_{z z}^{u}(t)=\varepsilon_{0}-\dot{\varepsilon}_{z z}^{u} \cdot t=\varepsilon_{0}\left(1-\frac{t}{T_{0}}\right) \\
\sigma_{z z}^{u}(t)=E \cdot \varepsilon_{z z}^{u}(t)=-\sigma_{a}\left(1-\frac{t}{T_{0}}\right)
\end{array}\right.
$$

where $E=2(1+v) \rho c_{s}^{2}$ is the Young's modulus, $\varepsilon_{z z}^{u}(t)$ and $\dot{\varepsilon}_{z z}^{u}$ are, respectively, the axial strain and axial strain rate at time $t$.

Based on Eq. 4 and the following boundary condition

$$
\left.\Delta \sigma_{z z}^{u}(t)\right|_{t=0}=0
$$

the evolution of the additional stress corresponding to $0<t \leq T_{0}$ can be expressed as

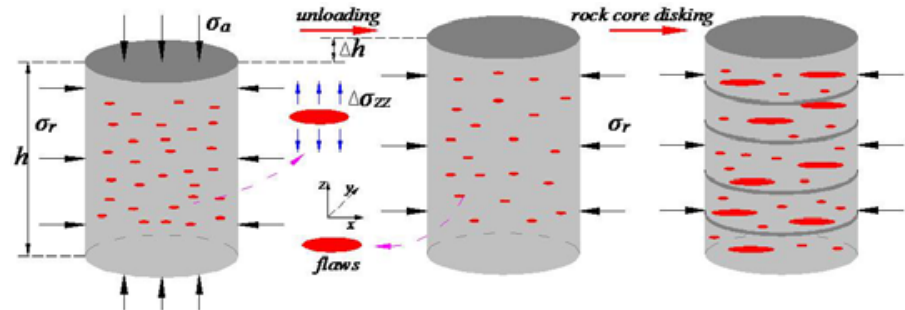

(a)

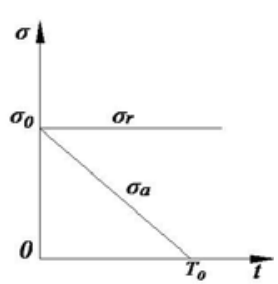

(b)

Fig. 2: (a) Triaxial unloading test: cylindrical specimen with initial hydrostatic stress; (b) abrupt unloading mode: the confining pressure is kept constant while the axial stress is reduced rapidly; in the end, the core disking occurs. 


$$
\Delta \sigma_{z z}^{u}(t)=-\frac{1}{3} \frac{k l \sigma_{a}}{\eta T_{0}}\left(1-e^{-\frac{\eta}{l} t}\right)
$$

at the end of the unloading process, i.e., at time $T_{0}, \sigma_{a}=0$ and $\dot{e}_{i j}=0$; then Eq. 3 can be rewritten as

$$
\frac{\mathrm{d} \Delta \sigma_{i j}}{\mathrm{~d}\left(t-T_{0}\right)}=-\frac{\eta}{l} \Delta \sigma_{i j}, \quad\left(t>T_{0}\right)
$$

Considering the continuity of stress, the solution of Eq. 8 is

$$
\Delta \sigma_{z z}^{u}(t)=-\frac{1}{3} \frac{k l \sigma_{a}}{\eta T_{0}}\left(1-e^{-\frac{\eta}{l} T_{0}}\right) e^{-\frac{\eta}{l}\left(t-T_{0}\right)}
$$

Substituting Eqs. 5, 7, and 9 into Eq. 2, the expression of the local stress can be expressed as

$$
\begin{cases}\sigma_{z z}^{\mathrm{loc}}(t)=\sigma_{a}\left(1-\frac{t}{T_{0}}\right)-\frac{1}{3} \frac{k l \sigma_{a}}{\eta T_{0}}\left(1-e^{-\frac{\eta}{l} t}\right) & \left(0 \leq t \leq T_{0}\right) \\ \sigma_{z z}^{\mathrm{loc}}(t)=-\frac{1}{3} \frac{k l \sigma_{a}}{\eta T_{0}}\left(1-e^{-\frac{\eta}{l} T_{0}}\right) e^{-\frac{\eta}{l}\left(t-T_{0}\right)} & \left(t>T_{0}\right)\end{cases}
$$

The local stress has direct effect on the stress field in the rock, especially the stress field around the flaw. Equation 10 shows that the local stress is influenced by many factors, such as the flaw scale $l$ and the unloading duration $T_{0}$.

\section{PARAMETERS' EFFECTS ON ROCK CORE DISKING}

\subsection{Influence of flaw scale}

In this section, in order to investigate the flaw scale's effect on the local stress, the parameters are as follows: $T_{0}=100 \mathrm{~s}, \sigma_{0}=50 \mathrm{MPa}, \eta=$ $2 \times 10^{-8} \mathrm{~m} / \mathrm{s}, \quad l=1 \times 10^{-6} \sim 1 \times 10^{-3} \mathrm{~m}$; the curves of the local stresses are obtained according to Eq. 10 .

Figures 3 and 4 show that, with the other conditions kept unchanged, the larger the flaw scale, the larger the maximum local tensile stress is. In addition, for flaws with the same scale, the maximum local tensile stress corresponding to the shorter unloading duration is larger. For example, when $T_{0}$ $=100 \mathrm{~s}$, the maximum local tensile stresses corresponding to $l=1 \times 10^{-6} \mathrm{~m}$, $5 \times 10^{-6} \mathrm{~m}, 1 \times 10^{-5} \mathrm{~m}, 1 \times 10^{-4} \mathrm{~m}, 1 \times 10^{-3} \mathrm{~m}$ are about $\sigma_{z z \max }^{\text {loc }}=5,14,15,15$, $15 \mathrm{MPa}$, respectively, but when $T_{0}=400 \mathrm{~s}$, the corresponding maximum lo- 


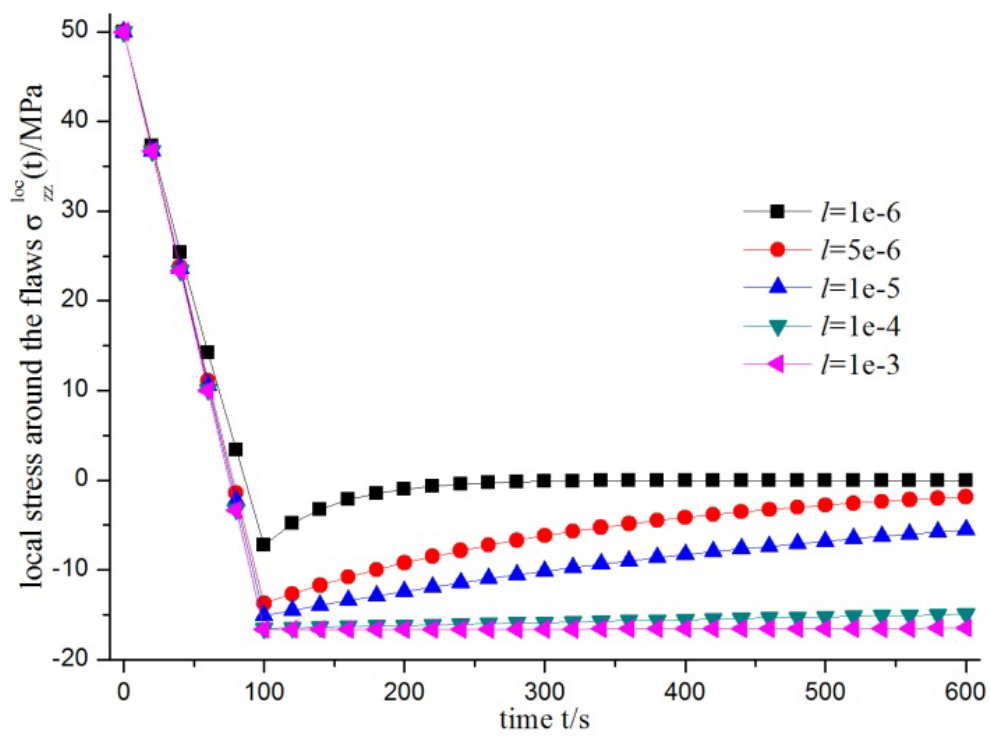

Fig. 3. Time-history curves of the local stresses (corresponding to $T_{0}=100 \mathrm{~s}$ ).

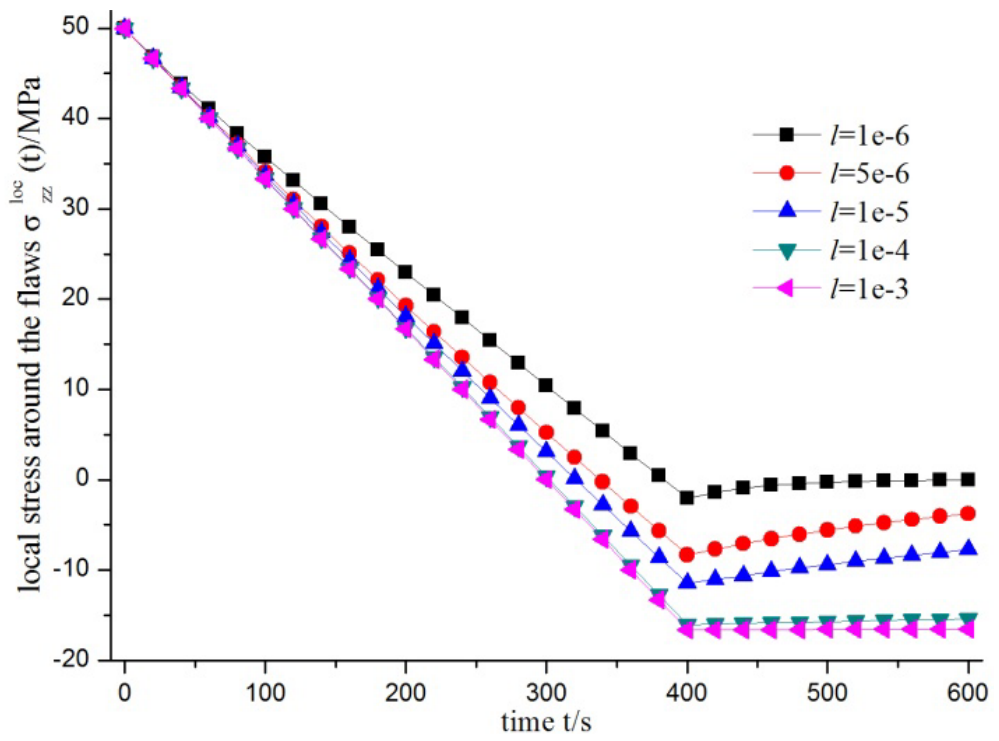

Fig. 4. Time-history curves of the local stresses (corresponding to $T_{0}=400 \mathrm{~s}$ ).

cal tensile stresses are about $\sigma_{z z \max }^{\text {loc }}=0,5,10,15,15 \mathrm{MPa}$, respectively. The increase of the unloading duration will obviously decrease the local tensile stresses which are caused by the flaws with smaller scales, such as $l=1 \times 10^{-}$ ${ }^{6} \mathrm{~m}, 5 \times 10^{-6} \mathrm{~m}, 1 \times 10^{-5} \mathrm{~m}$. So, the unloading duration will directly influence the stress field in the rock. 
We can also see that, when $t=0$, the local stresses are in compressive mode, but when $t=T_{0}$ the local stresses are in tensile mode. The phenomenon that during the unloading process the local stresses will change from compression to tension is a new finding in this paper; we can infer that core disking may occur when the local tensile stress is greater than the specimen's tensile strength. Therefore, it seems reasonable to establish a new criterion for rock core disking based on this finding.

\subsection{Relations between the flaw scale and the maximum local tensile stress}

The analysis above shows that for flaws with the same scale, the shorter unloading duration can cause a larger local tensile stress. If the unloading duration is too long, there is almost no local tensile stress around a smallscale flaw, but there is a local tensile stress around it if the unloading duration is decreased. It seems that more flaws can be "activated" under the short unloading duration (or at high unloading rate). In this paper, we regard the core disking as a result of the situation in which the local tensile stress exceeds the tensile strength. Thus, in order to activate more flaws, a short unloading duration is needed, which coincides well with the test phenomenon that the core disking occurs at a high unloading rate. The relations between the flaw scales and the local tensile stresses are shown in Fig. 5.

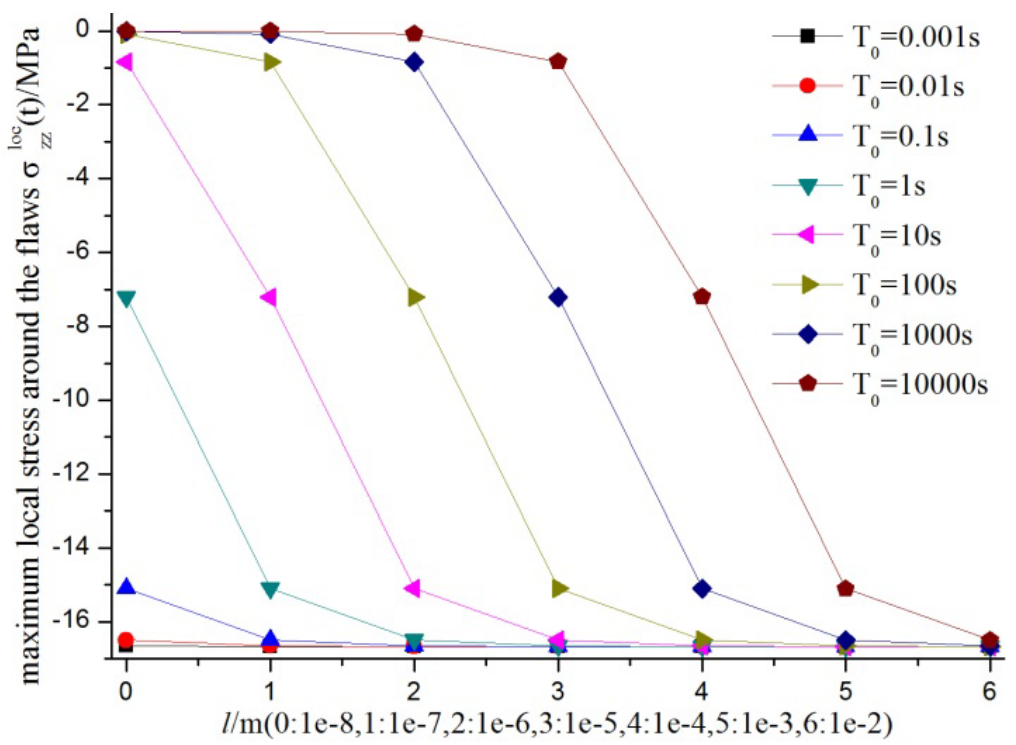

Fig. 5. Relations between the flaw scales and the maximum local tensile stresses during the unloading process (the numbers on the $x$-axis corresponding to scales $l=1 \times 10^{-8} \sim 1 \times 10^{-2} \mathrm{~m}$ are $0 \sim 6$, respectively). 
Figure 5 shows that if $T_{0}=10000 \mathrm{~s}$, there is no local tensile stress around the flaw whose scale is less than $l=1 \times 10^{-5} \mathrm{~m}$, i.e., in this situation, only the flaw whose scale is greater than $l=1 \times 10^{-5} \mathrm{~m}$ can be activated. In order to be activated, the flaw scales corresponding to the unloading durations $T_{0}=10000 \mathrm{~s}, 1000 \mathrm{~s}, 100 \mathrm{~s}, 10 \mathrm{~s}$ must be greater than $l=1 \times 10^{-5} \mathrm{~m}$, $l=1 \times 10^{-6} \mathrm{~m}, l=1 \times 10^{-7} \mathrm{~m}, l=1 \times 10^{-8} \mathrm{~m}$, respectively. For any flaw scale, the local tensile stress corresponding to the higher unloading rate is greater than that corresponding to the lower unloading rate. The range of the activated flaw scale decreases obviously with the increase of the total unloading duration from $0.001 \mathrm{~s}$ to $10000 \mathrm{~s}$. For example, when $T_{0} \leq 0.01 \mathrm{~s}$, all the flaws given in Fig. 5 can be activated, but when $T_{0} \geq 0.1 \mathrm{~s}$, only a part of the given flaws can be activated. Besides, when the unloading duration is short enough, the local stress would not increase as the unloading duration is decreased. For example, the local stresses are almost the same under the conditions of $T_{0}=0.001 \mathrm{~s}$, and $T_{0}=0.01 \mathrm{~s}$. It can be inferred that core disking would not take place when the unloading rate is low, may be because: (i) the number of the activated flaws isn't large enough; (ii) the local tensile stress caused by the activated flaw corresponding to the low unloading rate is not large enough to exceed the tensile strength.

\subsection{Influence of unloading duration}

In this section the parameters are as follows: the initial stress $\sigma_{0}=50 \mathrm{MPa}$, $l=1 \times 10^{-6} \mathrm{~m}, \eta=2 \times 10^{-8} \mathrm{~m} / \mathrm{s}, k=1, T_{0}=0.1 \mathrm{~s} \sim 400 \mathrm{~s}$. The curves obtained according to Eqs. 7, 9, and 10 are presented in the next figures.

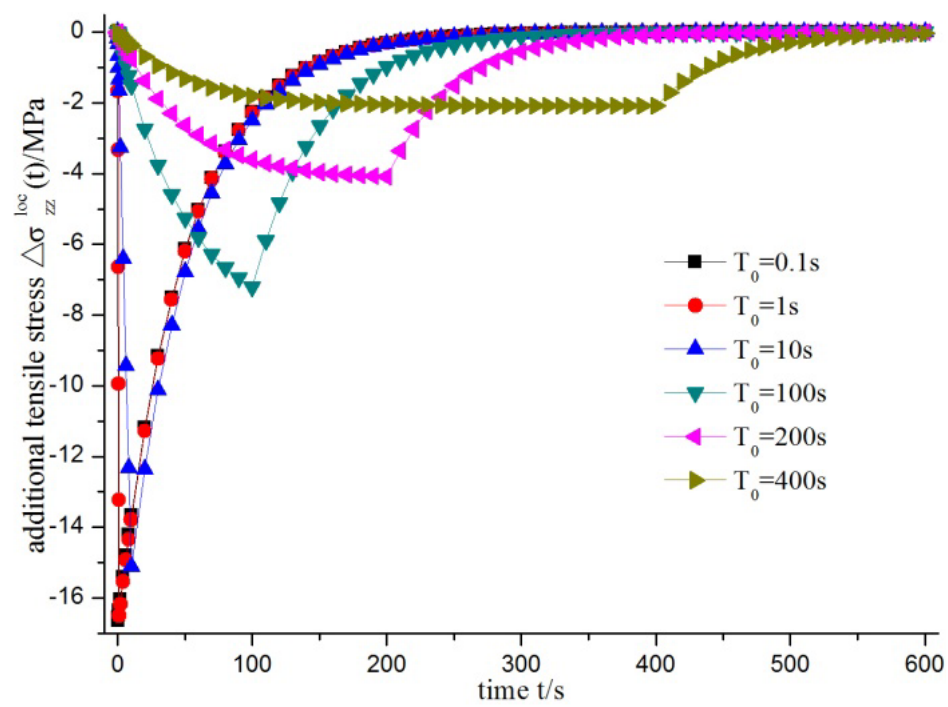

Fig. 6. Time-history curves of the additional tensile stresses. 


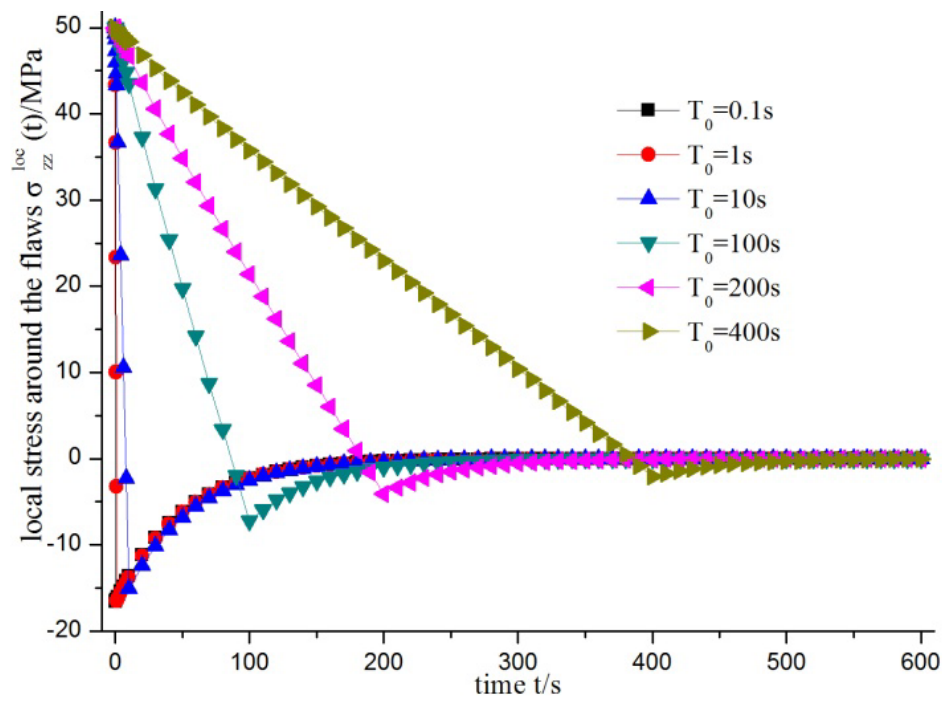

Fig.7. Time-history curves of the local stresses.

Figures 6 and 7 show that the shorter the unloading duration $T_{0}$, the larger the additional stress and the local tensile stress will be. The local stresses are compressive at $t=0$ while tensile at $t=T_{0}$, but the additional stresses are tensile during the whole process of unloading. Both the additional tensile stress and the local tensile stress reach their maximum values at $t=T_{0}$ and decrease to zero in the end. Besides, the local tensile stresses corresponding to $T_{0}=0.1 \mathrm{~s} \sim 10 \mathrm{~s}$ are all about $17 \mathrm{MPa}$, which are greater than the tensile strength of many kinds of rocks.

Considering that there are large numbers of flaws in the rock, and compared to the small-scale flaw, the large flaw can cause a large local tensile stress; therefore, we can infer that there must be so many flaws around which the local tensile stress is greater than $17 \mathrm{MPa}$. Therefore, it is reasonable to attribute the core disking to the exceedance of a local tensile stress over the tensile strength.

\section{CRITERION FOR CORE DISKING AND ITS TEST}

\subsection{New criterion for rock core disking}

During the unloading process, the local tensile stress will reach its maximum value at time $T_{0}$, assume that core disking takes place when the maximum local tensile stress reaches the crack initiation stress $\sigma_{c i}$, but considering that the determination of $\sigma_{c i}$ is complex, we replace it with the tensile strength $\sigma_{p}$, and then the criterion for rock core disking presented in this paper can be expressed as 


$$
\sigma_{z z}^{l o c}\left(t=T_{0}\right) \geq \sigma_{p} .
$$

According to Eq. 10, we obtain the initial hydrostatic stress $\sigma_{0}$ for rock core disking

$$
\sigma_{0} \geq \frac{3}{k} \sigma_{p}
$$

Considering that $0.5 \leq k \leq 3$ (Qi and Qian 2009), the initial stress for rock core disking can be expressed as

$$
\sigma_{0} \geq 6 \sigma_{p}
$$

Equation 13 shows that, for the cylindrical specimen with initial hydrostatic stress $\sigma_{0}$, under the triaxial unloading test, the core disking is likely to occur if the initial stress is more than six times the tensile strength.

In fact, based on our analysis, the initial hydrostatic stress $\sigma_{0}$ in Eq. 10 can be substituted by the axial stress $\sigma_{a}$, and the radial stress seems to have no obvious effect on core disking. If the maximum principal stress is in the axial direction of the specimen, then according to Eq. 13, the maximum principal stress $\sigma_{1}$ for rock core disking is

$$
\sigma_{1} \geq 6 \sigma_{p}
$$

In the following part of this paper, the presented criterion which is expressed by Eqs. 13 and 14 is tested both by the laboratory tests and the existing data from the published resources.

\subsection{Laboratory test of the criterion}

\subsubsection{Obtaining the tensile strength of the red sandstone by using the Bra- zilian test}

The specimens with no visible flaws were all cored from the same large rectangular block and in the same orientation. The cylindrical specimens are about $25 \mathrm{~mm}$ in height and $50 \mathrm{~mm}$ in diameter, in accordance with the ASMT standard. The data obtained from our test are listed in Table 1.

The data shows that the tensile strength of the red sandstone is about 5.0 $\mathrm{MPa}$; substituting $\sigma_{p}=5.0 \mathrm{MPa}$ into Eqs. 13 and 14 , the range of the initial hydrostatic stress and the maximum principal stress for core disking is

$$
\begin{gathered}
\sigma_{0} \geq 30 \mathrm{MPa}, \\
\sigma_{1} \geq 30 \mathrm{MPa} .
\end{gathered}
$$

Equations 15 and 16 show that, for the red sandstone, during the unloading process of the axial stress, the core disking is most likely to occur in one 
Table 1

Sizes of the specimens and data obtained from the Brazilian tests

\begin{tabular}{|c|c|c|c|c|}
\hline No. & $\begin{array}{l}\text { Diameter } \\
{[\mathrm{mm}]}\end{array}$ & $\begin{array}{l}\text { Height } \\
{[\mathrm{mm}]}\end{array}$ & $\begin{array}{c}\text { Bearing capacity } \\
{[\mathrm{KN}]}\end{array}$ & $\begin{array}{c}\text { Tensile strength } \\
{[\mathrm{MPa}]}\end{array}$ \\
\hline 1 & 49.1 & 25.4 & 7.11 & 3.63 \\
\hline 2 & 49.1 & 25.4 & 10.22 & 5.22 \\
\hline 3 & 49.1 & 25.2 & 9.83 & 5.06 \\
\hline 4 & 49.1 & 25.3 & 11.00 & 5.64 \\
\hline 5 & 49.1 & 25.3 & 6.35 & 3.26 \\
\hline 6 & 49.1 & 25.2 & 10.21 & 5.26 \\
\hline 7 & 49.1 & 25.1 & 9.01 & 4.66 \\
\hline 8 & 49.1 & 25.3 & 7.03 & 3.60 \\
\hline 9 & 49.1 & 25.2 & 7.84 & 4.04 \\
\hline 10 & 49.1 & 25.2 & 7.20 & 3.71 \\
\hline 11 & 49.1 & 25.1 & 7.56 & 3.91 \\
\hline 12 & 49.1 & 25.3 & 8.52 & 4.37 \\
\hline 13 & 49.1 & 25.3 & 11.02 & 5.65 \\
\hline 14 & 49.1 & 25.2 & 11.01 & 5.67 \\
\hline 15 & 49.1 & 25.2 & 11.90 & 6.13 \\
\hline 16 & 49.1 & 25.4 & 9.00 & 4.60 \\
\hline 17 & 49.1 & 25.1 & 8.89 & 4.59 \\
\hline 18 & 49.1 & 25.3 & 10.43 & 5.35 \\
\hline \multicolumn{4}{|c|}{ Average value of the tensile strength } & $4.92(\approx 5.0)$ \\
\hline
\end{tabular}

of the following two cases: (i) the initial hydrostatic stress is greater than $30 \mathrm{MPa}$; (ii) the maximum principal stress is in the axial direction and is greater than $30 \mathrm{MPa}$.

\subsubsection{Simulating the rock core disking under the critical hydrostatic stress and maximum principal stress}

The unloading tests conducted by Bauch and Lempp (2004) with the red German sandstone are good examples for our test; the sizes of the specimens used in their tests are $70 \mathrm{~mm}$ in diameter and $140 \mathrm{~mm}$ in height. Two grain sizes are adopted, one is medium and the other is fine. The tensile strengths of the two specimens are about 3.9 and $4.9 \mathrm{MPa}$, respectively. When the core disking occurs, the measured minimum initial hydrostatic stresses for the two specimens are, respectively, about 20 and $25 \mathrm{MPa}$, the ratios of the initial hydrostatic stress to the tensile strength are, respectively, 5.13 and 5.10, which are close to our theoretical prediction of 6 . 


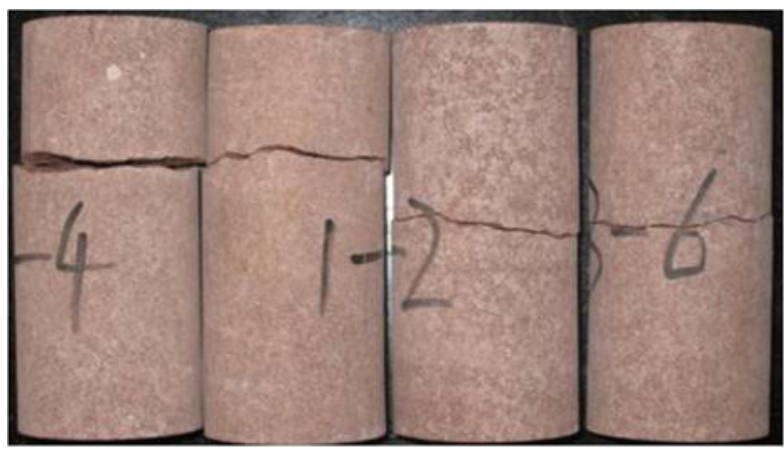

(a)

(b)

(c)

(d)

Fig. 8. Unloading core disking: the initial stresses from left to right are: $\begin{array}{llll}\text { (a) } \sigma_{r}=\sigma_{a}=25 \mathrm{MPa}, & \text { (b) } \sigma_{r}=20 \mathrm{MPa}, \sigma_{a}=25 \mathrm{MPa}, & \text { (c) } \sigma_{r}=\sigma_{a}=30 \mathrm{MPa} \text {, }\end{array}$ (d) $\sigma_{r}=20 \mathrm{MPa}, \sigma_{a}=30 \mathrm{MPa}$, respectively.

Based on their tests and our prediction, the sizes of the specimens adopted in our test are also $70 \mathrm{~mm}$ in diameter and $140 \mathrm{~mm}$ in height, and the initial stresses chosen for our tests are as follows
(a) $\sigma_{r}=\sigma_{a}=25 \mathrm{MPa}$,
(b) $\sigma_{r}=20 \mathrm{MPa}, \sigma_{a}=25 \mathrm{MPa}$,
(c) $\sigma_{r}=\sigma_{a}=30 \mathrm{MPa}$,
(d) $\sigma_{r}=20 \mathrm{MPa}, \sigma_{a}=30 \mathrm{MPa}$,

where 20,25, and $30 \mathrm{MPa}$ are, respectively, about 4, 5, and 6 times the tensile strength; both according to our analysis and the tests conducted by Bauch and Lempp (2004), core disking is impossible to occur if the initial stress is only 4 times the tensile strength. In the abrupt mode, core disking occurs under the given stress conditions and the phenomena observed in our test are shown in Fig. 8.

Figures $8 \mathrm{a}$ and $8 \mathrm{c}$ show that the core disking can occur if the initial hydrostatic stresses are, respectively, 5 and 6 times the tensile strength. Figures $8 \mathrm{~b}$ and $8 \mathrm{~d}$ show that if the axial stresses are, respectively, 5 and 6 times the tensile strength, the core disking can occur although the radial stress is less than the critical stress. Thus, we can deduce that the initial hydrostatic stress for rock core disking is about 5 to 6 times the tensile strength. Under the non-hydrostatic stress condition, core disking can occur in the situation in which the maximum principal stress is in the axial direction and is about 5 to 6 times the tensile strength.

\subsection{Data from published resources to test our prediction}

As mentioned above, in the abrupt mode, the ratios of the initial hydrostatic stress to the tensile strength for rock core disking reported by Bauch and 
Lempp (2004) are of about 5.13 and 5.10, respectively. Considering that the rock core disking is simultaneously influenced by factors such as the unloading duration, the local tensile stress and the test conditions, besides, the internal structure of the rock is also far more complex; therefore, we can infer that when ignoring these factors, our prediction is acceptable. Thus, the initial hydrostatic stress $\sigma_{0}$ for rock core disking is $\sigma_{0} \geq(5 \sim 6) \sigma_{p}$.

In addition, Lim and Martin (2010) also reported their statistical results of rock core disking observed in the Underground Research Laboratory, Canada. Their study shows that the initiation of core disking is controlled by the maximum principal stress, core disking is most likely to initiate if the maximum principal stress $\sigma_{1}$ is about 6.5 times the tensile strength $\sigma_{p}$, i.e., $\sigma_{1} / \sigma_{p} \geq 6.5$. Considering that the distribution of the in situ stress around the URL may be irregular and the drilling direction in their tests may not always parallel to that of the maximum principal stress, besides, the statistical results are only based on granite, therefore, our prediction is close to their statistical results, and we can infer that core disking is most likely to occur if the maximum principal stress is greater than six times the tensile strength, i.e., $\sigma_{1} \geq 6 \sigma_{p}$.

\section{CONCLUSIONS}

Rock core disking is a time-dependent tensile failure phenomenon. This paper reveals that during the unloading process, there are local tensile stresses caused by the flaws; the expression for the local tensile stress is derived, and the effects of both the flaw scale and the unloading duration on the local tensile stress are analyzed. A new criterion for rock core disking is presented and the following conclusions are drawn.

- In the abrupt unloading mode, there is local stress in the axial direction of the cylindrical specimen and the local stress will change from compressive to tensile during the process. Both the additional tensile stress and the local tensile stress will reach their maximum values at the end of the unloading process.

- In proper initial stress state, the core disking can occur only at the high unloading rate, which may be due to the following reasons: (i) the high unloading rate can activate more flaws; (ii) at the high unloading rate, the local tensile stress caused by any activated flaw is large.

口 In this paper, the premises of both our theoretical prediction and laboratory test are that there is no visible flaw in the hard rock. At present, our prediction has only been tested by the red sandstone; therefore, in order to extend its application to other kinds of hard rocks, further experimental study is required. 
- At high unloading rate, core disking is most likely to occur under the following two stress conditions: (i) the initial hydrostatic stress is greater than 5 to 6 times the tensile strength, i.e., $\sigma_{0} \geq(5 \sim 6) \sigma_{p}$; and (ii) the maximum principal stress is greater than 6 times the tensile strength, i.e., $\sigma_{1} \geq 6 \sigma_{p}$.

Acknowledgements. The authors would like to express their sincere gratitude to the financial support by the National Key Basic Research Program of China (Grant No. 2013CB036005), National Natural Science Foundation of China (Grant No. 51527810); in addition, their appreciation also goes to the editor and the anonymous reviewers for their comments.

\section{References}

Almeida, L.C.R., E.A. Vargas Jr., and R.P. Figueiredo (2006), Mechanical characterization of rock splitting planes in granitic rocks, Int. J. Rock Mech. Min. Sci. 43, 7, 1139-1145, DOI: 10.1016/j.ijrmms.2006.03.014.

Bauch, E., and C. Lempp (2004), Rock splitting in the surrounds of underground openings: an experimental approach using triaxial extension test. In: R. Hack, R. Azzam, and R. Charlier, Engineering Geology for Infrastructure Planning in Europe, Springer, Berlin, 244-254.

Corthesy, R., and M.H. Leite (2008), A strain-softening numerical model of core disking and damage, Int. J. Rock Mech. Min. 45, 3, 329-350, DOI: 10.1016/ j.ijrmms.2007.05.005.

Ishida, T., and T. Saito (1995), Observation of core discing and in situ stress measurements; Stress criteria causing core disking, Rock Mech. Rock Eng. 28, 3, 167-182, DOI: 10.1007/BF01020150.

Jaeger, J.C., and N.G.W. Cook (1963), Pinching-off and disking of rocks, J. Geophys. Res. 68, 6, 1759-1765, DOI: 10.1029/JZ068i006p01759.

Lempp, C. H., and H.B. Muhlhaus (1985), Splitting and core disking in deep boreholes. In: 2nd Int. Symp. on Observation of the Continental Crust through Drilling, 94.

Li, Y.Y., and D.R. Schmitt (1998), Drilling-induced core fractures and in situ stress, J. Geophys. Res. 103, B3, 5225-5239, DOI: 10.1029/97JB02333.

Lim, S.S., and C.D. Martin (2010), Core discing and its relationship with stress magnitude for lac du bonnet granite, Int. J. Rock Mech. Min. 47, 2, 254264, DOI: 10.1016/j.ijrmms.2009.11.007.

Matsuki, K., N. Kaga, T. Yokoyama, and N. Tsuda (2004), Determination of three dimensional in situ stress from core discing based on analysis of principle 
tensile stress, Int. J. Rock Mech. Min. Sci. 41, 7, 1167-1190, DOI: 10.1016/ j.ijrmms.2004.05.002.

Obert, L., and D.E. Stephenson (1965), Stress conditions under which core disking occurs, Soc. Min. Eng. Trans. 232, 3, 227-235.

Qi, C.Z., and Q.H. Qian (2009), Basic Problems of Dynamic Deformation and Fracture of Rock Mass, Science Press, Beijing (in Chinese).

Rodionov, V.N., and I.A. Sizov (1982), Appearance of nonuniformity of the stressed state as a result of fracture of rocks, J. Min. Sci. 17, 4, 323-331, DOI: 10.1007/BF02497255.

Rodionov, V.N., and I.A. Sizov (1989), Model of a rigid body with dissipative structure for geomechanics, Soviet Min. 24, 6, 491-501, DOI: 10.1007/ BF02498606.

Stacey, T.R. (1982), Contribution to the mechanism of core disking, J. S. Afr. Inst. Min. Metall. 82, 9, 269-274.

Zhu, W.-S., W.-M. Yang, X.-J. Li, X. Lu, and D.-J. Yu (2014), Study on splitting failure in rock masses by simulation test, site monitoring and energy model, Tunn. Undergr. Sp. Tech. 41, 152-164, DOI: 10.1016/j.tust.2013.12.007.

Received 28 July 2015

Received in revised form 14 December 2015

Accepted 12 January 2016 